\title{
Design of Quadriplex Extrusion Line Control System Based on ControlNet
}

\author{
Chen Mingxia \\ Guangxi scientific experiment center of mining, metallurgy and environment \\ Mechanical and control engineering college \\ Guilin University of Technology, China \\ wjunt@sohu.com
}

\begin{abstract}
With the development of electronic technology, Fieldbus control network is applied in industrial control system widely. In CGEC Company, Fieldbus technology is commonly used in electric control system of machine. ControlNet is one kind of Fieldbus control network. This paper briefly introduces the characteristics and basic working principle of ControlNet, describes the control system of quadriplex extrusion line based on ControlNet with examples, which is composed of ControlLogix5000 series PLC, Flex I/O remote station with ControlNet interface, Panel View Plus touch panel, PowerFlex70 series inverter, etc. The practical experience and consequence of several project witness that the networks based on ControlNet fieldbus technology have apparent advantages such as simple structure, stable technical performance, reducing the amount of wiring that is why it is convenient to commissioning and maintenance, enhancing the flexibility and reliability of the system in the best way, and so on. In conclusion, the control system base on the ControlLogix5000 series PLC+ControlNet fiedbus network is a good choice in the project that needs high system performance.
\end{abstract}

Keywords- quadriplex extrusion line;fieldbus; ControlNet; touch panel; flex I/O; inverter)

\section{INTRODUCTION}

With the rapid development of social economy and science technology, in rubber industry, the requirement of equipments for quality and quantity is increasing, and rubber machinery and equipment is moving in large-scale, linkage, automatic, intelligent direction. CGEC extrusion equipments, one of the company's main series of products have been from the original single extruder to produce various products of many varieties of composite extrusion machine. At present, the company's duplex, triplex, quadriplex extrusion lines have been widely used in the tire industry at home and abroad. And the quadriplex extrusion line is in the domestic original, the leading proprietary technology products. Due to the requirement for the mechanical properties of complicated and highly automated equipment, intelligentize, matching followed complicated control system and control requirements are higher. At the same time, the current control technology with a variety of electronic products in the field of automatic control in high-speed development period, product update very quickly. And based on various aspects of demand, like production, statistics, analysis, management, maintenance and so on, the user to the functional requirement of the control system and stability requirements is also becoming more and more high, especially some senior customers, in addition to the system is stable and reliable, also need to collect, record amounts of data and information. Therefore, with the development of the industrial field bus technology, as its simple, reliable, economical and practical, and excellent communication function etc, the control system based on the bus network is gradually spreading into some important area and applied in different field of industry control. However, how to synthesize many special factors in the current situation of the variety types of products and field bus to design a suitable control system and meet the needs of different levels, is what engineers must take into account, such as user needs, cost factors, and the system performance factors.

\section{FIELDBUS APPLICATION IN CGEC COMPANY}

At present, according to the different needs of different users, the types of bus network control systems are often used in CGEC company's projects have the following kinds: Siemens S7 series PLC+ Profibus-DP bus network control system , Mitsubishi's Q series PLC + CC - Link bus network control system, Rockwell company ControlLogix5000 series PLC+ControlNet bus network control system.

Just as we all know that PROFIBUS started earlier, it is one of the most successful field buses in current, and has been widely used. It has good openness and major automation manufacturers have the support of Profibus-DP products. However, there is a puzzling problem that as the increase of the stations on network and the length of it, its stability will be reduced, especially in the case of EMC does not attach enough consider when in the situation of installation, the system will be more communication problems and then leading to downtime; It will take longer time for technician to solve the problem because of its diagnostic information is vague. So, it is very important to pay special attention to the network distribution and EMC requirements when dealing with the system planning and designing.

Network cc-link EMC requirements are not strict as Profibus-DP network, also its stability is better than Profibus-DP under harsh environment, PLC system configuration and network cost is lower, but its 
compatibility is poor, many well-known brands of automation products haven't CC - Link network interface, and Mitsubishi PLC programming control software visibility is poorer, diagnostic information is lack, programming is tricky, and address is needed to calculation.

Based on ControlLogix5000 series PLC+ControlNet fieldbus network control system has high cost compared with the previous two, but the response speed and stability of the ControlNet are better. Field test results and application experience in multiple projects show that it will not recur problem in supposing that the first time communication can connected according to the requirement of each connection joints, and to the can't connect site, PLC software can also give accurate diagnostic information. Programming model of RS5000 programming software and connection address is very convenient and the diagnostic information is complete, which to the debugging and the maintainer is definitely good news. While such system had both advantages and disadvantages. The openness of the system is not very good, and the software distinguish much slimly and variety, for the first time user will feel more difficult, often encounter software is not compatible module or adapter card then need additional processing, which create enormous spans of wasted time. But on the whole, the performance of the system than the Profibus-DP network system and the CC-Link network system is better.

The following content, setting the quardriplex extrusion line as an example, to elaborate the practical application of network control system based on ControlNet fieldbus.

\section{CONTROL SYSTEM OF QUADRIPLEX EXTRUSION LINE BASE ON CONTROLNET NETWORK}

\section{A. The Introduction for Characteristics of ControlNet}

ControlNet based on the modified CanBus technique, it is a high-speed and definitive industrial control network accord with IEC61158 Type 2 standard, used to establish an explicit, predictable, and repeatable communication between industrial equipment. Its data transfer rate of $5 \mathrm{Mb}$, supports defined and repeatable scheduled communications data exchange, also supports unscheduled communication. It is primarily designed for the communication between PLC, I/O devices, Programming terminals, as well as the operation of terminal. As an independent network, it can be used for I/O control, message sending, programming, and terminal operation. Medium access method makes full use of the advantages of the producer-consumer model, supports multiple consumers, multi-cast, and point to point communication. And support the Controllogix redundancy and PLC-5 ControlNet hot standby (efficient system redundancy processors). All use of the coaxial cable of the distance can be up to $6 \mathrm{~km}$, on the same link support 99 nodes at most and have 64 connections, the longest distance between two nodes up to $1000 \mathrm{~m}$, the distance of 48 node can up to 250m; Communication distance can be up to scores of kilometers after using optical fiber and repeater, The network structure can use bus, tree and star structure or combination structure. The user can extend the physical length according to the need of increasing node Numbers or improve safety performance. ControlNet is a network based on the bus, so a terminal resistance must be installed at the end of each segment.

As ControlNet network is in data link layer, so information is transmitted through establish connections between the nodes. Accessing information from Network media is controlled by the unique time limit access algorithm, namely to adopt concurrent time domain multiple access (CTDMA) method, in each control node of the network (NUI) for the refresh interval transmit information. We can set by the software of millisecond network refresh time (the NUT) to configuration the frequency of NUI repetition, the scope of the NUT for $2 \mathrm{~ms}$ to $100 \mathrm{~ms}$. The NUT can be divided into three parts: the scheduled time and unscheduled time, the maintenance time. Scheduled time: each scheduled node (Based on the sequential order in a loop) to ensure there is a chance to transfer information. Unscheduled time: all the nodes in an order to transmit information circulation. Maintenance time: reserved for network maintenance time, using the address information transmitted by the lowest node to ensure the synchronization of other nodes.

First, confirm that you have the correct template for your paper size. This template has been tailored for output on the US-letter paper size. If you are using A4-sized paper, please close this template and download the file for A4 paper format called "CPS_A4_format".

\section{B. Hardware Configuration of the Control System}

Quadriplex extrusion line mainly includes four feeders, four screw extruders, hydraulic head, temperature control units, parts I of downstream, parts II of downstream, calender, skiver and so on. Considering the servo control of skiver is suitable to the nearest, to place the master station of PLC control system in the control cabinet beside skiver, and then according to the characteristics of the other input and output control points of different parts and quantity, give full play to the superiority of collecting and distributing system, remote I/O stations of PLC according to the following parts to take consider : 1.Feeder NO.1; 2.Feeder NO.2; 3.Feeder NO.3; 4.Feeder NO.4; 5.Temperature control units NO.1; 6.Temperature control units NO.2; 7.Hydraulic station; 8.The left side of head; 9.The right side of head; 10.The main operation desk; 11.Cabinets on platform; 12.Calender control cabinet; 13.Calender NO.1 operation box; 14. Calender NO.2 operation box.

1) Master station of PLC: Consisting of Rockwell company ControlLogix5000 series PLC, using 10 slot rack, the type of CPU is1756-L62, the EtherNet module is used for communication to managing main computer system and touch panels, two ControlNet modules are used to form the networks to connect to the remote $\mathrm{I} / \mathrm{O}$ stations and other devices, a servo control module is used to control two servo drive through the optical fiber optical fiber connection, then according to the I/O characteristics and number on skiver and accessories parts to configurative corresponding analog and digital I/O modules. This series PLC modules are high performance and intelligent I/O modules: support the producerconsumer model, the I/O module produce information only when needed, thus relieving the burden of processor, 
improve the efficiency of the system; All I/O without hardware setup, but through software configuration, easy to rapid configuration $\mathrm{I} / \mathrm{O}$ system; And all modules support hot-plugging.

2) Remote I/O stations : Choose Rockwell Company's 1794 Flex series products to construct various PLC remote I/O stations, each substation consist by a 1794ACN15 ControlNet adapter and all kinds of I/O modules match each station control points. Each 1794 - ACN15 adapter can contain eight modules at most, through which the above two small button switch can set ControlNet station address, address range from 01 to 99 , and show the light on the power supply and communication diagnosis. 1794 Flex I/O is a flexible, high cost performance, and is suitable for distributed applications modular I/O.

3) DC driver : It adopts CT company's MENTOR II series DC driver to control extruders and calenders. Since it has not ControlNet network adapter, it is controlled by CPU of PLC via connection with the Flex I/0 directly.

4) AC inverter: It adopts Rockwell Company's inverter series of PowerFlex70 to control the operation and speed of all transducer motors in the whole line. Each inverter has the network adapter card that with ControlNet. It can set the ControlNet address that between 01 to 99 by two micro-knob switch. Network adapter card with ControlNet is used to realize the data exchange with the CPU, it also has the function of receive the control instruction from $\mathrm{CPU}$ and send the status information.

5) AC servo drive: This system consists of two sets of Ultra3000 series servo drivers from Rockwell Company, they communicate to the servo module 1756 - M03SE on master station of PLC by optical fiber. This system is used to control two servo motors and other parts of skiver, then to implement the fixed-length cutting function in accordance with the requirements of products formula.

6) Touch panel: There is a touch panel on the master operating station and the cutting operation box for each. The type of touch panel is PanelView Plus series products bundied a communication interface of EtherNet in Rockwell Company. The system is bundied a network module of EtherNet because of PLC and upper management computer are needed to pass the communication of EtherNet. So, it chooses to connect PLC with touch panel by EtherNet. It makes contribution to cost saving, reduce the number of sites of ControlNet and improve the speed of communication.

System networks configuration is shown in Fig .1.

\section{System Configuration in RSLogix5000}

1) The system configuration: When creating a new project and writing the program in PLC software for control system, first of all is to establish a corresponding project in RSLogix5000 software and system configuration, its configuration process steps are as follows: a).In the new application I/O Configuration, choose the type of the CPU of PLC by the right mouse button according to design drawings, select the software version number, and give a name for the CPU, choose the corresponding rack slot number. b).A backplane labels of CPU added appears in the PLC project program after added CPU, by right-click the labeling, add the type of I/O modules, ControlNet modules, and Ethernet modules according to the design drawings. And pay attention to select the slot number of module and give a name for the module. c)After adding ControlNet module, then add the drive or I/O modules of substation, right-click ControlNet module, choose the type of module according to drawings. d).For components that are not belong to $\mathrm{AB}$ company products, if it is hung on the ControNet module, you can choose ControlNet-MODULE, and then carried out in accordance with the characteristics of the device configuration. When finish the configuration the structure as shown in Fig .2. 


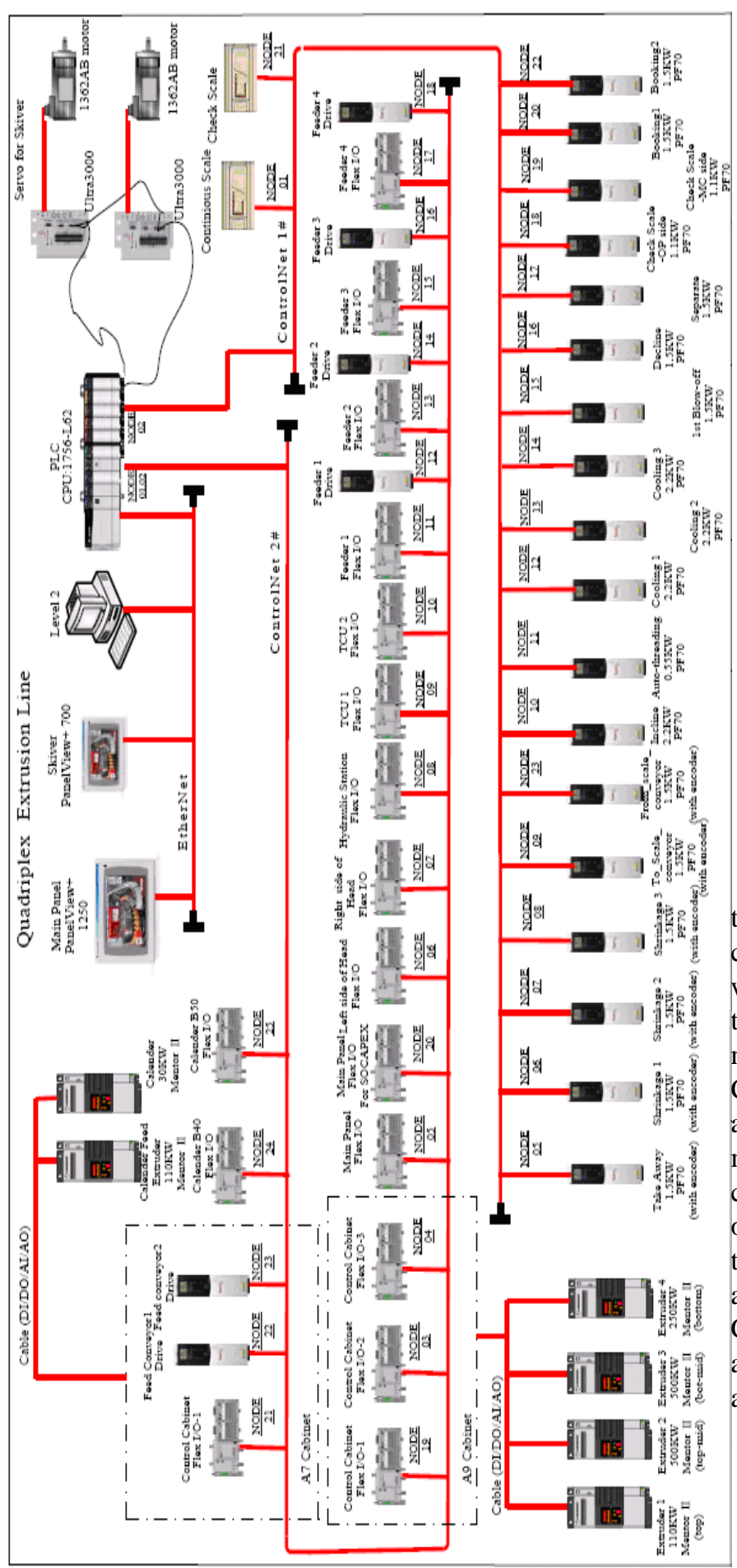

Figure 1. The networks configuration of control system.

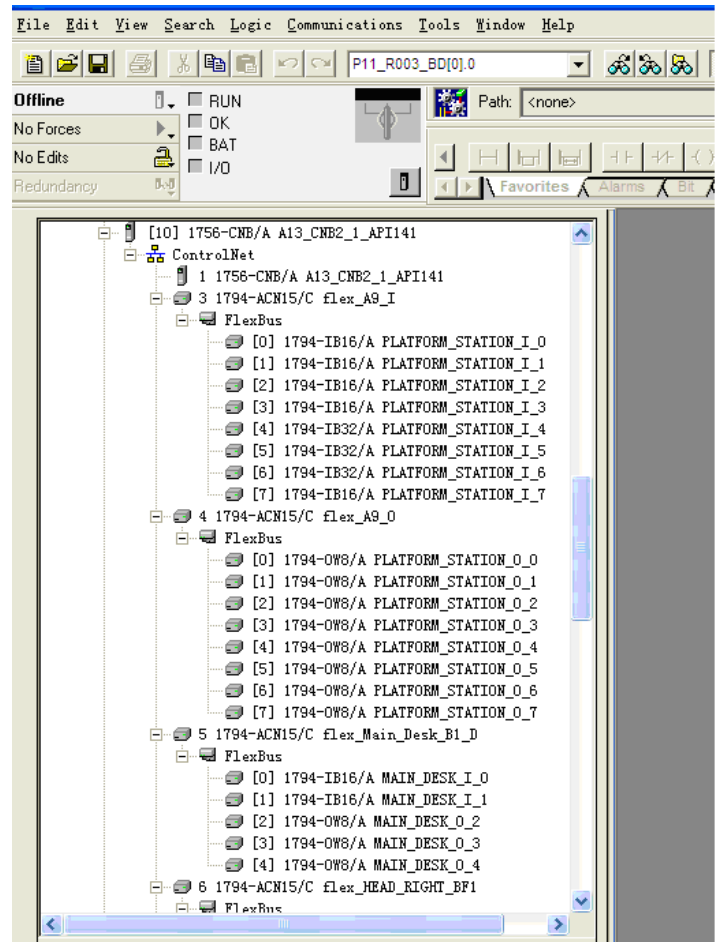

Figure 2. PLC system configuration.

2) Methods to schedule the ControlNet Network: First to confirm all hardware units on network are configured correctly and the communication is normal. Pilot lamps will flash or turn to red if there is any problem. There are two ways that can be chosen to schedule ControlNet network--To schedule by creating a new file of ControlNet and to schedule by ControlNet module that is added in the configuration of PLC hardware. The second method as follows: a). Right-click the ControlNet module, choose the Schedule The ControlNet Network in the third option RSNetWorx, as shown in Fig .3. b). Click "OK" then the program automatically open RSNetworx software, and scan of network nodes online. c). Edit enabled: Choose the Edits Enable in the picture, software automatically schedules. d).Save the file, file path will appear in the features of ControlNet module.

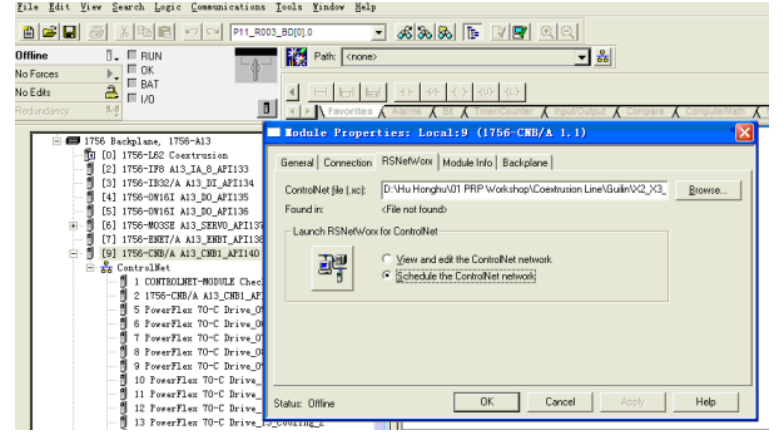

Figure 3. Network schedules.

After finishing schedule ControlNet network, there will appear yellow warning signs if the hardware configuration module is abnormal, you can check the state of module, and schedule the network again after the faults were removed. 


\section{Design of Software for Control System}

1) The structure of PLC program: The structure of PLC program: Main structure of PLC program for quadriplex extrusion line as shown in Fig .4. Each relatively independent part of the control system there is same structure form in PLC program. The Routine number and its specific application are different according to the requirements of their respective functions within a subroutine. An example about division of the program structure forms as shown in Fig .5.

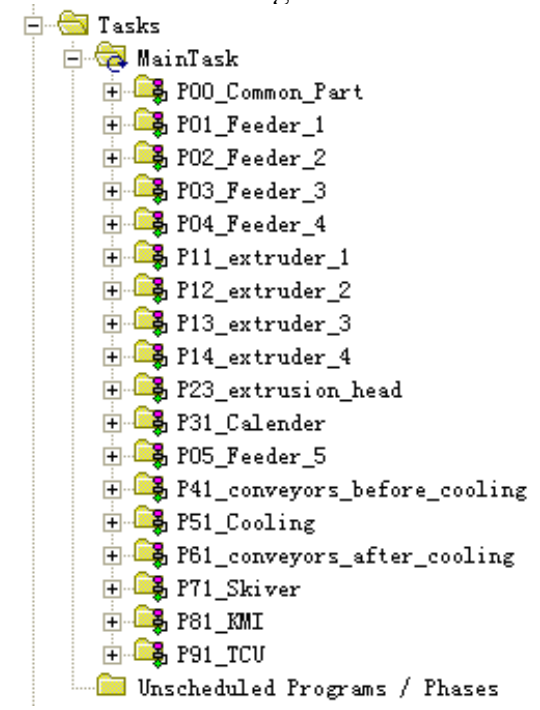

Figure 4. Main structure of PLC Program.

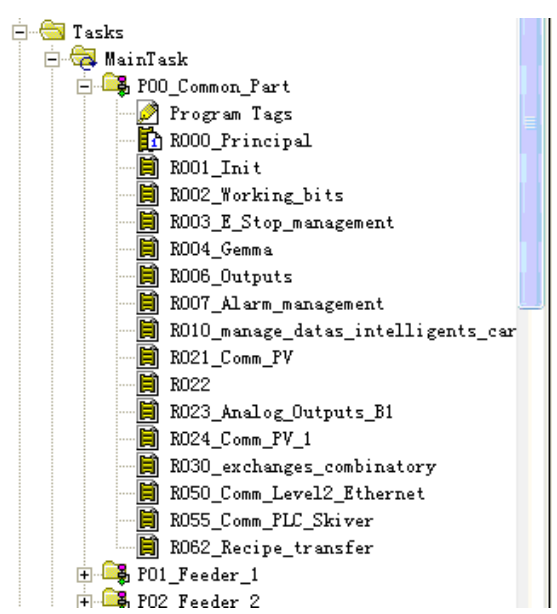

Figure 5. The division of the Program structure.

As shown in Fig .4 and Fig .5, each part can be made relatively independent programs group in a catalogue which similar to the folder in the condition of RsLogix 5000 platform. It is irrelevant when using the same name in this directory of local label and other program directory, so that we can copy similar parts program what only to modify its global tags and signal correlation, so can greatly reduce the workload of programming. For programming software of RsLogix 5000 use the tag (label) programming method, so that the users is free to prepare the label name, type and quantity, even make the label name briefly, easy to understand, also make different people can understand the programs easily that written by others, thus forming standardization programs according to each company's own requirements. There is a very prominent advantages for programming method of using the tag, that is the users can program even the project system configuration don't finish (as the module of the system input and output channels has not yet to determine its specific function), just match the tag with a specific module in software channel corresponding after the completion of system configuration, without having to modify the program again. This method of programming completely changed the former programming methods: programmer programming must base on drawing design and each module functions of input and output channels have finished, otherwise have to modify the program again. In this way, the project design and programming can be carried out at the same time, as well as less time consuming. It is convenient to inspect the information of components have disposed the in the whole Controllogix system, such as the PLC modules and the drive components, this not only make the system of information collection is more easy to implement, but also greatly facilitate the debugging and maintenance personnel.

2) Design of touch panel interface: The design of the touch panel interface with all kinds of screen and functions is carried out according to the requirements from equipment, production technology and user. Touch panel interface designed by RSView software of Rockwell, then correlate touch panel with CPU according to the actual network path in the software, and match the variables between touch panel and PLC program, and final generate the runtime file and download it to the touch panel, so the functions of each screen will be realized according to the scheme designed. It is unnecessary adding another tabs to connect with ControlLogix 5000 CPU in the label, but directly read the tabs of ControlLogix 5000 CPU after establishing communications with ControlLogix 5000 system. So that simplifies the programming of touch panel.The user interface of this system mainly includes main monitoring screen, the user login (password) management screen, monitoring screens of components, recipe management screens, equipment parameter setting screens, service screens, alarm information screens and so on. The function of switching each other between screens is realized by the function of user login and transformation button given in each screen. The main screen of two touch panels as shown in Fig .6 and Fig .7 respectively.

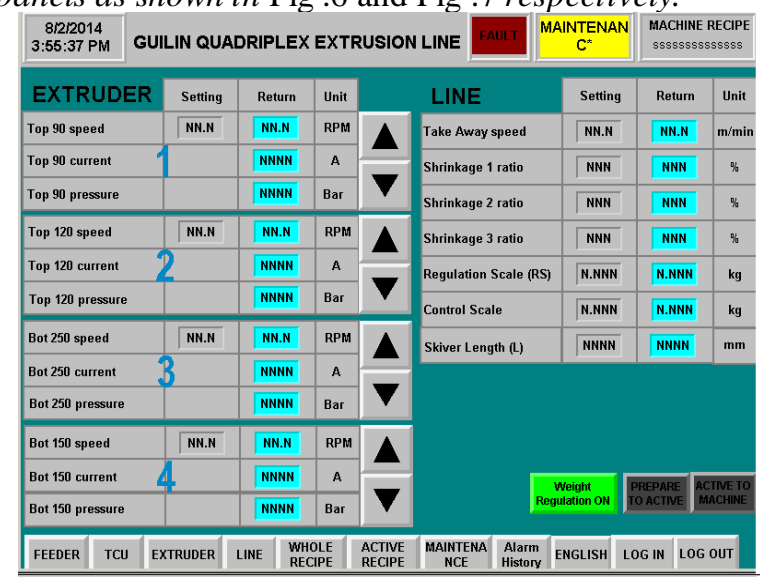

Figure 6. The main screen of whole line. 


\begin{tabular}{|c|c|c|c|c|c|}
\hline \multicolumn{2}{|l|}{$\begin{array}{l}\text { 4:11:09 PM } \\
\text { 8/2/2014 }\end{array}$} & SKIVER & FAULT & AUTO & $\begin{array}{l}\text { RUN RECIPE } \\
\text { sssssssssssssss }\end{array}$ \\
\hline \multicolumn{2}{|c|}{ SAFETY POWER NOT READY } & Setting & Return & Unit & \\
\hline \multicolumn{2}{|c|}{ Product length (final) } & NNNN & NNNN & $\mathrm{mm}$ & \\
\hline \multicolumn{2}{|c|}{ Product length (L-recipe) } & NNNN & & $\mathrm{mm}$ & \\
\hline \multicolumn{2}{|c|}{ Product length (N-recipe) } & NNNN & & $\mathrm{mm}$ & \\
\hline \multicolumn{2}{|c|}{ Product length offset (PV) } & NNNN & & $\mathrm{mm}$ & \\
\hline \multicolumn{2}{|c|}{ Product length offset (L-rec) } & NNNN & & $\mathrm{mm}$ & DEFAULT \\
\hline \multicolumn{2}{|c|}{ Product length offset (N-rec) } & NNNN & & $\mathrm{mm}$ & \\
\hline \multicolumn{2}{|c|}{ Measure Belt Speed } & & NN.N & $\mathrm{m} / \mathrm{Min}$ & \\
\hline \multicolumn{2}{|c|}{ Decline Belt Speed } & & NN.N & m/Min & \\
\hline & & & & & LOG ON \\
\hline RETURN & & GRAFCET & DIAGNOSTICS & $\begin{array}{l}\text { ALARM } \\
\text { HISTORY }\end{array}$ & MAINTENANCE \\
\hline
\end{tabular}

Figure 7. The main screen of skiver.

\section{CONCLUSION}

The practical experience and consequence of several project witness that the networks which based on ControlNet Field bus technology have apparent advantages in the following. First, its structure is simple. Second, its technical performance is stable. Third, it will reduce the amount of wiring, and that is why it is convenient to commissioning and maintenance. Last but not least, it enhances the flexibility and reliability of the system in the best way. However, the functions of good to program, parameter setup and read, and failure diagnosis and so on, which not only make the program to achieve the highly modularization and standardization easily, but make debugging and maintaining faster in RSLogix5000 software platform. Its function and speed are better than some other Fieldbus networks. Based on the series of ControlLogix 5000, the control system base on the series of ControlLogix5000 PLC+ ControlNet fiedbus network is a good choice in the program of highly system performance.

\section{REFERENCES}

[1] Baidu library, ControlLogix composition and the application of PLC control system

[2] Rockwell Automation.ControlLogix System User Manual. . 2011

[3] Rockwell Automation. RSLogix5000 Programming Software Product Profile. . 2011.

[4] Rockwell Automation.RSLinx User Manual. . 2011.

[5] Rockwell Automation.RSLogix5000 User Manual. . 2011.

[6] Yuzhu Mi, motion control system based on ControlLogix platform application [J];Science and technology information;22, 2012.

[7] Fanjin Sun,Weiming Tong.The Studying of Virtual Token Passing Approach in ControlNet. High Technology . 2004.

[8] Marceclo Luis Dultra.Fieldbus Control System. Advances in Instrumentation and Control . 2001

[9] Lan Jianpeng, "Analysis and comparison of the system of automatic control based on ControlNet bus," Science and Technology Innovation Herald, 2013,(9).doi: 10.3969/j.issn.1674098X.2013.09.029

[10] Rockwell Automation. RsnetWorx for ControlNet Online Help. . 